\title{
Synthesis and fluorescence characteristics of ATP-based FRET probes
}

\begin{abstract}
Norman Hardt, $\neq$ Stephan M. Hacker $\neq$ and Andreas Marx*
Adenosine triphosphate (ATP) analogues labelled with two dyes suitable for undergoing Förster Reso nance Energy Transfer (FRET) have the potential to be valuable tools to continuously study the enzymatic activity of ATP consuming enzymes. Here, we present a synthesis strategy that allows obtaining these ATP analogues in a straight forward manner. Earlier studies indicate that modifying ATP at the O2' and the $\gamma$ position is a very promising starting point for the design of these probes. We synthesized probes modified with five different combinations of dyes attached to these positions and investigated their fluorescence characteristics in the non cleaved state as well as after enzymatic hydrolysis. All presented probes largely change their fluorescence characteristics upon cleavage. They include ratiometric FRET probes as well as dark quenched analogues. For typical in vitro applications a combination of the sulfonated polymethine dyes Sulfo Cy3 and Sulfo Cy5 seems to be most promising due to their excellent solubility in aqueous buffer and a large change of fluorescence characteristics upon cleavage. For this combination of dyes we also synthesized analogues modified at the $\gamma$ and the $C 2$ or the $03^{\prime}$ position, respectively, as these attachment sites are also well accepted by certain ATP consuming enzymes. These analogues show comparably large changes in fluorescence characteristics. Overall, we present new ATP based FRET probes that have the potential to enable monitoring the enzymatic activity of ATP consuming enzymes.
\end{abstract}

\section{Introduction}

Many enzymatic systems use ATP, for example to drive metabolic processes thermodynamically uphill, to couple chemical free energy to mechanical, electrical, or photoelectrical events or to transduce extracellular signals into intracellular processes. ${ }^{1}$ Understanding these different enzymes and their way of regulation can largely help to understand and to modify various biological pathways.

ATP consumption can be monitored using various assays in vitro. Monitoring the cleavage products of ATP can be performed using radioactive ATP analogues ${ }^{2}$ or detecting the released phosphate or pyrophosphate using colorimetric detection. ${ }^{3}$ Both methods require further processing of the enzymatic reaction prior to measurement and, therefore, do not allow continuous detection of ATP turnover from a single

Department of Chemistry, Konstanz Research School Chemical Biology, University of Konstanz, Universitätsstr. 10, 78457 Konstanz, Germany.

E mail: Andreas.Marx@uni konstanz.de; Fax: +49 753188 5140; Tel: +497531885139

$\ddagger$ These authors contributed equally. enzymatic reaction. Alternatively, an enzyme-coupled assay can be used to monitor ATP turnover. ${ }^{4}$ In this assay, ADP produced during the enzymatic reaction can be converted to ATP by pyruvate kinase with the help of phosphoenolpyruvate (PEP). The pyruvate formed is reduced by $\mathrm{NADH}$ to form lactate and NAD, whose absorption is used to determine the amount of ATP consumed. Enzyme-coupled assays can be monitored continuously, but have the disadvantage that effects on the additional enzymes used have to be considered when studying effectors of ATP consuming enzymes.

We have recently described an assay to directly monitor ATP cleavage by the ubiquitin activating enzyme UBA1 in the process of ubiquitin activation. ${ }^{5}$ For this assay, a fluorescence donor and a fluorescence acceptor that are suitable for undergoing Förster Resonance Energy Transfer (FRET) are attached to the same ATP analogue (Fig. 1). Upon cleavage by the enzyme of interest the two fluorophores are separated, resulting in a large change in fluorescence characteristics. This change can be easily detected using fluorescence spectroscopy. The assay enabled us to directly study various effects on the ubiquitin activation by UBA1 in real-time without the use of additional enzymes or reagents that may also be affected.

UBA1 belongs to the rather small family of ubiquitin-like protein activating enzymes ${ }^{6}$ and thus, it would be of great interest to transfer this concept to other ATP consuming 


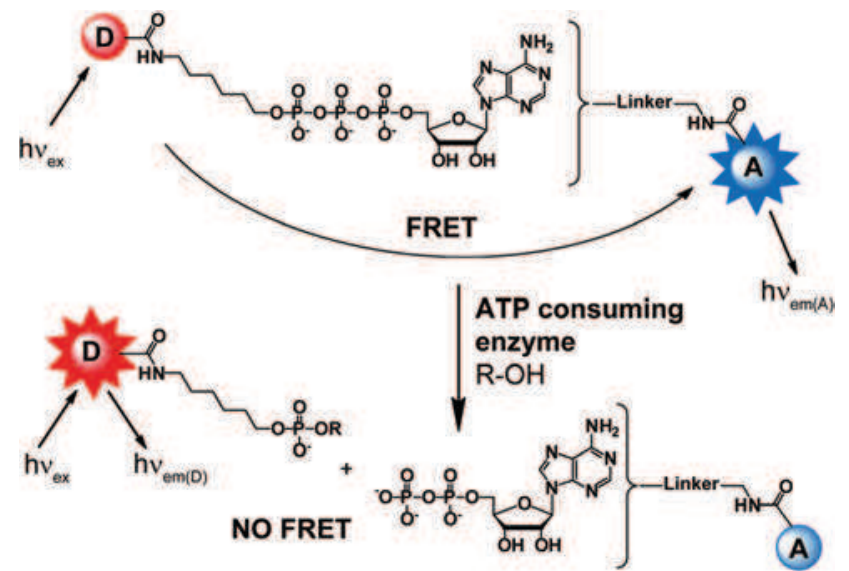

Fig. 1 Concept of doubly labelled ATP analogues. A fluorescence donor (D) is attached to the $\gamma$ phosphate of ATP and, upon excitation, transfers its energy intramolecularly to a fluorescence acceptor (A) attached to the same molecule of ATP. After cleavage this energy transfer is no longer possible, leading to a large change in fluorescence characteristics.

enzymes. Modification at the N6-position of ATP as used to study UBA1 is not accepted by many of these enzymes. ${ }^{7,8}$ In contrast, analogues modified at the $O 2$ '-position of the ribose have been shown to be widely accepted by ATP consuming enzymes. ${ }^{8,9}$ Furthermore, modification at the ${ }^{2} 3^{\prime}$ - and the C2-position is beneficial in certain cases. $^{8}$ However, their photophysical properties are elusive.

In this work, we present the synthesis of several new doubly labelled ATP analogues as activity probes for nucleotide dependent enzymatic reactions. The modifications were appended to positions $\mathrm{O2}^{\prime}, \mathrm{O}^{\prime}$ ', and $\mathrm{C} 2$, respectively, and the $\gamma$-phosphate of the phosphate chain of ATP. For the latter attachment, we synthesized phosphate esters as they were reported to be stable under the employed conditions, thus avoiding non-enzymatic hydrolysis of the probes. ${ }^{10}$ To obtain these molecules, we elaborated on a versatile synthesis strategy that enabled us to synthesize various probes in a straight-forward manner. Thereby we focused on polymethine dyes ${ }^{11}$ as they have been shown to be very well suited for FRET applications at short donor acceptor distances as used here. ${ }^{12}$ To test fluorescence characteristics of the dye combinations in the context of doubly labelled ATP analogues, we utilized the phosphodiesterase I from C. adamanteus (Snake Venom Phosphodiesterase, SVPD), which is known to cleave the $\alpha / \beta$ anhydride bond of many different nucleotides, as a model enzyme and recorded the fluorescence spectra after treatment with or without SVPD. ${ }^{5,13}$ These investigations revealed several ATP analogues that undergo significant changes of the fluorescence characteristics upon cleavage.

\section{Results and discussion}

\section{Synthesis of doubly modified ATP analogues}

First, we optimized the synthesis of doubly dye labelled ATP analogues. To be able to obtain different ATP analogues in a straight-forward manner, we constructed the doubly modified ATP scaffold with two orthogonally protected amines and introduced the dyes at the end of the synthesis sequence. In this way several doubly labelled ATP analogues can be obtained from one common scaffold. We thereby considered that the second amine has to be liberated in the presence of the first dye. Therefore, this deprotection reaction should be as mild as possible. We decided to use a trifluoroacetamide and an azide as amine precursors. First, the trifluoroacetamide can be deprotected under alkaline conditions and coupled with the first label. This leaves the azide completely unaffected. Finally, the azide can be reduced to the amine by the Staudinger reaction ${ }^{14}$ using very mild conditions ${ }^{15}$ and subsequently coupled to the second label.

To obtain the ATP scaffold modified at the $O 2^{\prime}$ - and the $\gamma$-position, we started with known triphosphate $1 .^{8}$ This molecule was transformed into its tetrabutylammonium salt, which can be used for alkylation with 6-azido-1-bromohexane at the $\gamma$-phosphate using an established method. ${ }^{10}$ Deprotection of the trifluoroacetamide was carried out using ammonia in water and the reaction mixture was purified using anionexchange chromatography and RP-HPLC. Anion-exchange chromatography was carried out as triphosphates that are not modified at the phosphate chain, like compound $\mathbf{1}$, tend to partially react to the corresponding di- and monophosphates in organic solvents as used for the alkylation reaction. These differently phosphorylated species are separated by anionexchange, which failed solely by RP-HPLC. This procedure resulted in $25 \%$ yield of ATP analogue 2, which already bears the first free amine ready for conjugation. ATP analogue 2, similar to all further ATP analogues, was obtained as its triethylammonium salt, which is soluble in water as well as in polar organic solvents and can be used for all further conversions.

To extend the scope of building blocks, we also synthesized the ATP analogue having the interchanged protecting group pattern, i.e. the azide at the $O 2$ '-position and the trifluoroacetamide at the $\gamma$-position (Scheme 1B). Therefore, we started with known $\mathrm{O}^{\prime}$-(6-azidohexyl)-adenosine ${ }^{8}$ and performed the triphosphate synthesis using a standard one-pot procedure. Purification by anion-exchange chromatography and RP-HPLC gave triphosphate 3 in a yield of $39 \%$. After transformation to its tetrabutylammonium salt, compound 3 was alkylated at the $\gamma$-phosphate using 1-iodo-6-trifluoroacetamido-hexane. Subsequently, the trifluoroacetamide group was cleaved and the reaction mixture purified using anion-exchange chromatography and RP-HPLC to give compound 4 in $25 \%$ yield over the last two steps. In this way, two doubly modified ATP analogues were obtained that are ready for the subsequent conjugation.

\section{Synthesis of doubly labelled ATP analogues}

Next, we set out to label the doubly modified ATP analogues with different combinations of dyes. Starting with compound 2 we performed the coupling with the Sulfo-Cy5 $N$-hydroxysuccinimide (NHS) ester in a mixture of DMF and sodium 


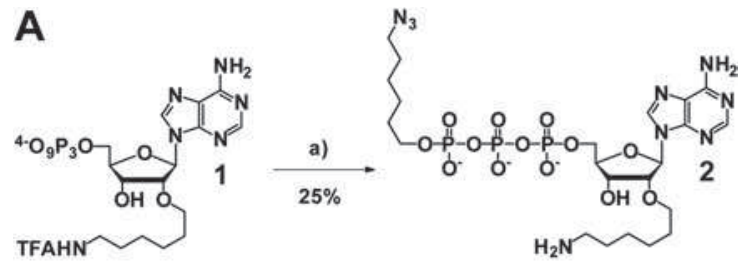

B
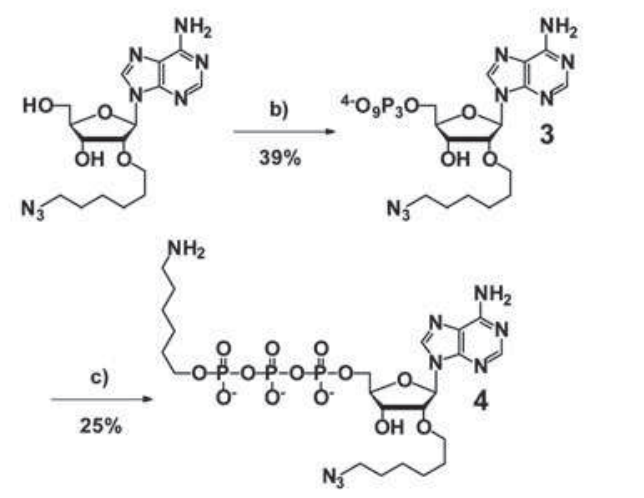

Scheme 1 Synthesis of modified ATP analogues. (a) (i) 6 Azido 1 bromo hexane, DMF, r.t., 12 h; (ii) ammonia in water, r.t. 4 h; (b) (i) $\mathrm{POCl}_{3}$, proton sponge, trimethyl phosphate, $0{ }^{\circ} \mathrm{C}, 1 \mathrm{~h}$; (ii) $\left(\mathrm{Bu}_{3} \mathrm{NH}^{+}\right)_{2} \mathrm{H}_{2} \mathrm{P}_{2} \mathrm{O}_{7}^{2}$, tributylamine, r.t., $30 \mathrm{~min}$; (iii) triethylammonium acetate buffer ( $\mathrm{pH}$ 7.5), r.t., $30 \mathrm{~min}$; (c) (i) 6 trifluoroacetamido 1 iodo hexane, DMF, r.t., 12 h; (ii) ammonia in water, r.t., $4 \mathrm{~h}$. All compounds were prepared as their triethylammonium salts.

carbonate buffer at pH 8.9 to yield compound $\mathbf{5}$ in $70 \%$ (Scheme 2A). Compound 5 and all further reactions were purified by RP-HPLC. Next, we performed the Staudinger reaction of the azide using TCEP in water, methanol and triethylamine at room temperature to provide compound 6 in $68 \%$ yield. This was followed by conjugation of the Sulfo-Cy3 NHS ester in $64 \%$ yield resulting in the final doubly labelled ATP analogue 7a. This corresponds to an overall yield of $30 \%$ over the last three steps. The same target molecule was also obtained starting from nucleotide 4 using an analogous synthesis scheme with reversed order of the conjugation of the two labels in a yield of $28 \%$ over the final three steps (Scheme 2B). Both methods of synthesis seem to be equally well suited to obtain the doubly labelled analogue.

Using this synthesis protocol we were able to attach four further combinations of dyes starting from compound $\mathbf{4}$. In this way we obtained a set of five different doubly labelled ATP analogues.

\section{Fluorescence characteristics of ATP analogues}

Having these compounds in hand we started to investigate their fluorescence characteristics. The ATP analogues were incubated with SVPD at $30{ }^{\circ} \mathrm{C}$ until complete cleavage was observed. Completion of the cleavage was monitored by analytical RP-HPLC (Fig. 2A-2C, ESI results, ESI Fig. S1-S7†). After incubation in the absence of SVPD only one compound, absorbing at the absorbance maximum of the donor, as well as at that of the acceptor, was observed in the RP-HPLC trace
(Fig. 2B). In contrast, after cleavage with SVPD two new peaks, absorbing only at the absorption maximum of the donor or the acceptor, respectively, were observed (Fig. 2C).

We tested three combinations of fluorescent donors with fluorescent acceptors. These FRET probes have the advantage that fluorescence intensity can change at two wavelengths. In this way, analysis of the ratio of the two fluorescence intensities allows monitoring cleavage independent of variations in probe concentration. All samples were adjusted to $1 \mu \mathrm{M}$ with $1 \times$ PBS and fluorescence spectra were recorded (Fig. 2D-2H).

First, we investigated the combination of Sulfo-Cy3 and Sulfo-Cy5 present in probe 7a (Fig. 2D, ESI Fig. S1†). In this case upon cleavage a large increase of Sulfo-Cy3 emission was detected, accompanied by a strong decrease in Sulfo-Cy5 fluorescence. The ratio of the two fluorescence intensities changes by a factor of approx. 140. This large change of fluorescence could be used for the very robust detection of ATP cleavage making this ATP analogue a very well suited ratiometric FRET probe.

Next, we turned to the combination of Sulfo-Cy5 and SulfoCy7 (probe 7b). These dyes have the advantage of fluorescence at even longer wavelengths. An approx. 37 -fold rise in fluorescence was detected for Sulfo-Cy5 upon cleavage (Fig. 2E, ESI Fig. S2†). In contrast, almost no acceptor fluorescence of Sulfo$\mathrm{Cy} 7$ fluorescence was detected in both states. This is probably due to the low fluorescence quantum yield of Sulfo-Cy $7^{11}$ and due to additional quenching effects in the non-cleaved probe. In this way, this analogue does not represent a useful ratiometric FRET probe.

Finally, we also investigated the combination of the nonsulfonated alternatives $\mathrm{Cy} 3$ and Cy5 in ATP analogue 7c (Fig. 2F, ESI Fig. S3†). These less polar dyes could be advantageous over their sulfonated analogues for cellular studies as they have the potential for passive cellular uptake. ${ }^{16}$ For the cleavage of this probe lower concentrations has to be used to avoid precipitation in the presence of magnesium ions. Using these conditions $>98 \%$ cleavage of the probe was obtained. Also in this case an increase of Cy3 emission and a decrease of Cy5 emission were detected. The ratio of fluorescence intensities changes 70 -fold. This probe is therefore also very promising for monitoring ATP consuming enzymes and could have advantages over probe 7a concerning cellular uptake. Nevertheless, as regards in vitro applications probe 7a has the advantage of higher solubility in aqueous buffer.

Additionally, we also investigated two ATP analogues having non-fluorescent acceptor dyes. These probes, although not ratiometric, could be well suited to study ATP cleavage as fluorescence turn-on probes. We chose to use the two fluorescence donors, Sulfo-Cy3 and Cy3, and combined these dyes with the Eclipse quencher ${ }^{17}$ that has well matching absorption characteristics for these two donors. ATP analogue 7d containing a combination of Sulfo-Cy3 and Eclipse quencher is soluble in water. Upon hydrolysis, the fluorescence intensity of Sulfo-Cy3 rises by a factor of 66 (Fig. 2G, ESI Fig. S4 $\dagger$ ). This makes this probe a very valuable tool to study ATP cleavage. 

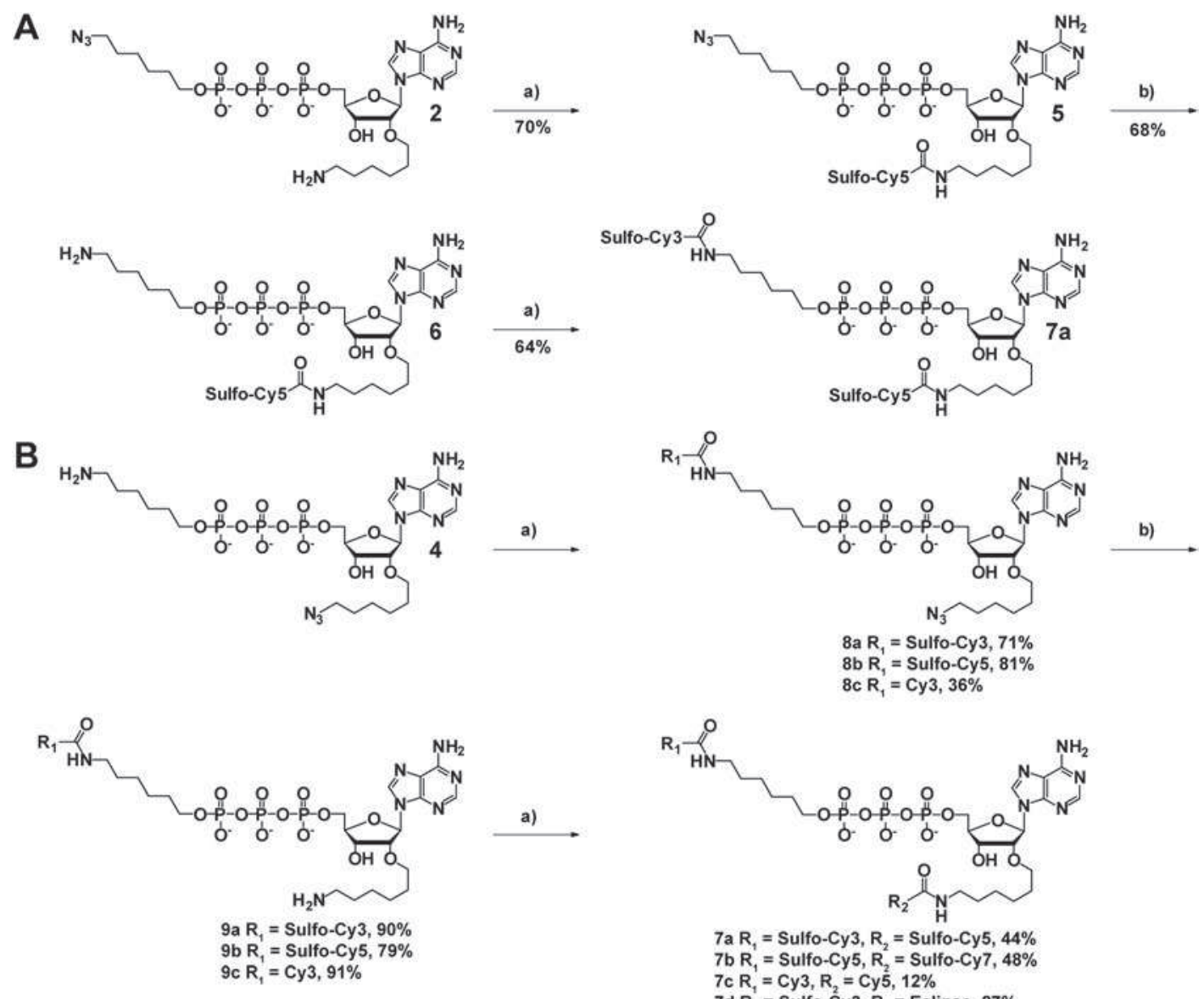

$7 \mathrm{a} R_{1}=$ Sulfo-Cy3, $R_{2}=$ Sulfo-Cy $5,44 \%$ $7 \mathrm{~b} R_{1}=$ Sulfo-Cy $5, R_{2}=$ Sulfo-Cy $7,48 \%$ $7 c R_{1}=C y 3, R_{2}=C y 5,12 \%$ $7 \mathrm{~d} R=$ Sulfo-Cy $3, R_{2}=$ Eclipse, $27 \%$
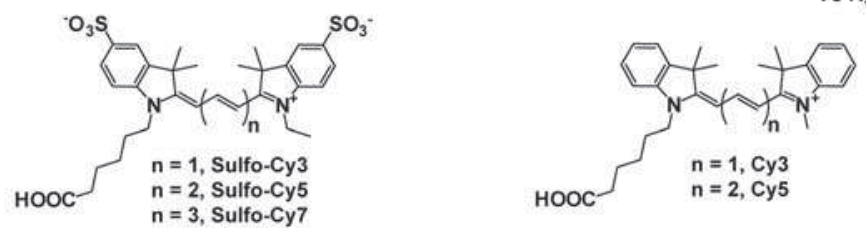
$7 \mathrm{e} R_{1}=$ Cy3,$R_{2}=$ Eclipse, $13 \%$

Scheme 2 Synthesis of doubly labelled ATP analogues. (a) NHS ester of the corresponding dye, DMF, carbonate buffer (pH 8.9 ), r.t., 2 12 h; (b) TCEP HCl, Et ${ }_{3} \mathrm{~N}$, $\mathrm{MeOH}, \mathrm{H}_{2} \mathrm{O}$, r.t. $412 \mathrm{~h}$. All compounds were prepared as their triethylammonium salts.

For the combination of $\mathrm{Cy} 3$ and the Eclipse quencher (probe 7e) large solubility problems in aqueous media containing magnesium were detected. At low concentrations and in the presence of $10 \%$ DMSO enzymatic hydrolysis of the probe by SVPD was performed yielding $>95 \%$ cleavage. Fluorescence spectra were obtained showing an approx. 90-fold increase of fluorescence intensity (Fig. 2H, ESI Fig. S5†). Nevertheless, due to the low solubility in aqueous buffer this probe is not well suited to study ATP consuming enzymes.

In summary, we present five different doubly labelled ATP analogues modified at the $O 2^{\prime}$-position and at the $\gamma$-phosphate that all show high changes of fluorescence characteristics upon hydrolysis. ATP analogues with two of these combinations of fluorescent dyes, namely Sulfo-Cy3 and Sulfo-Cy5 (7a) as well as Cy3 and Cy5 (7c) can be efficiently used as ratiometric FRET probes for ATP hydrolysis. Furthermore, the probe containing a combination of Sulfo-Cy3 with the fluorescence quencher Eclipse (7d) yields a dark-quenched FRET probe with very promising properties. $7 \mathbf{d}$ is almost nonfluorescent in the non-cleaved state and allows monitoring of ATP cleavage by a rise of fluorescence. Due to the high change of the ratio of fluorescence intensity and the very good solubility in water the combination of Sulfo-Cy3 and Sulfo-Cy5 can be considered best for typical in vitro applications. Nevertheless, probes like $\mathbf{7 c}$ and $\mathbf{7 d}$ may be advantageous for certain applications.

\section{Alternative attachment of the dyes}

To extend the scope of doubly labelled ATP analogues we also investigated alternative attachment sites to ATP. Here, we chose to use modifications at the $\mathrm{O3}^{\prime}$ - and the $\mathrm{C2}$-position as they are also well accepted by certain ATP consuming enzymes. $^{8}$ For this purpose, we decided to use the best 


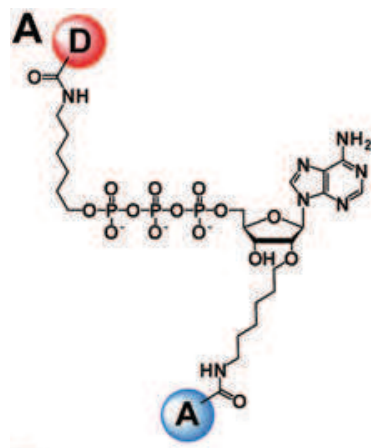

E

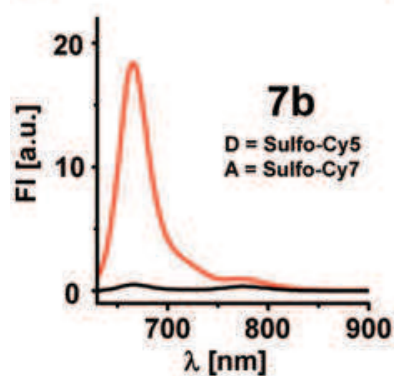

B

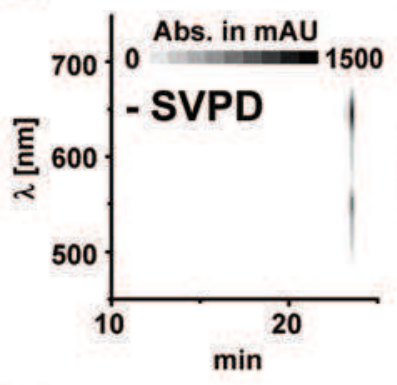

F

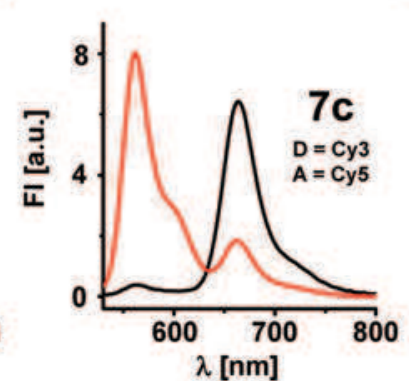

C

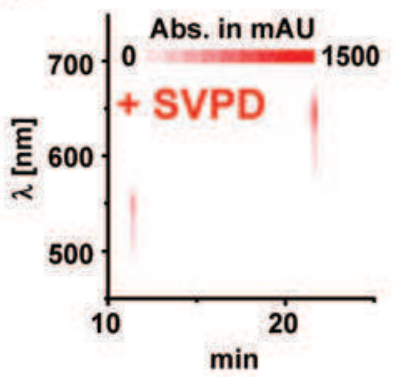

G

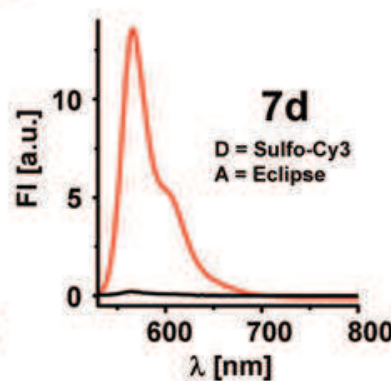

D

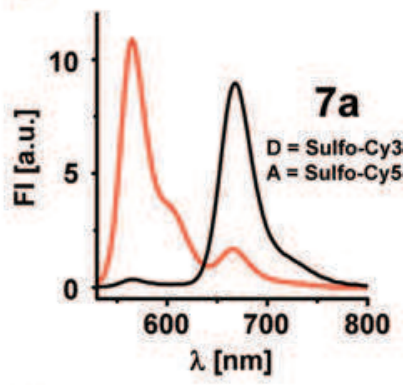

H

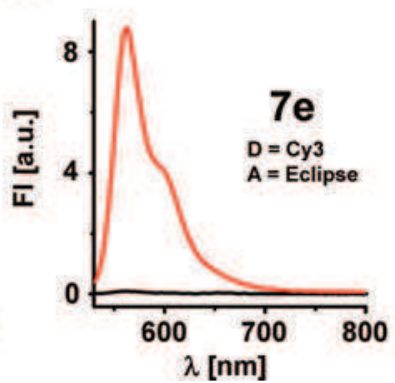

Fig. 2 Fluorescence characteristics of different doubly labelled ATP analogues. (A) General structure of ATP analogues labelled at the O2' and the $\gamma$ position with different dyes. D: fluorescence donor, A: fluorescence acceptor; (B) + (C) Exemplary RP HPLC analysis of probe 7a after treatment without ( SVPD) or with (+SVPD) the phosphodiesterase I from C. adamanteus. Abs.: absorbance at the indicated wavelength. All other RP HPLC analyses can be found in the ESI; + ( $D)$ ( $H$ ) Fluorescence spectra of compounds $7 \mathbf{a} \mathbf{7 e}$ after incubation without (black) or with (red) the phosphodiesterase I of $C$. adamanteus measured at a concentration of $1 \mu \mathrm{M}$ in $1 \times$ PBS with excitation at $510 \mathrm{~nm}(610 \mathrm{~nm}$ for probe $\mathbf{7 b})$.

combination of dyes leading to a ratiometric probe in the case of $\mathrm{O}^{\prime}$-modification, namely the dyes Sulfo-Cy3 and Sulfo-Cy5.

We started synthesizing the probe modified at the $C 2$-position and the $\gamma$-phosphate (Scheme 3A). Starting from known C2-(5-trifluoroacetamidopentynyl)-adenosine triphosphate ${ }^{8}$ we introduced the modification of the triphosphate at the $\gamma$-position by treatment with 1-bromo-6-azido-hexane as described above to provide the doubly modified triphosphate. Subsequently, the trifluoroacetamide protection group was cleaved by ammonolysis with ammonia in water to generate the free amine in compound $\mathbf{1 0}$ in $37 \%$ yield over two steps. The dye Sulfo-Cy5 was coupled using the method described above with a yield of 58\%. Next, the azide was reduced using TCEP generating the amine group of compound 12 in 66\% yield. Finally, Sulfo-Cy3 was introduced in an analogous fashion as before in $87 \%$ yield resulting in probe $\mathbf{1 3}$ doubly labelled at the $C 2$ - and the $\gamma$-position.

Next, we turned to the synthesis of the $03^{\prime}$ - and $\gamma$-labelled ATP analogue 17 (Scheme 3B). We started with the known O3'-(6-trifluoroacetamidohexyl)-adenosine triphosphate. ${ }^{8}$ Modification at the $\gamma$-phosphate using 1-bromo-6-azidohexane, followed by deprotection using aqueous ammonia gave doubly modified triphosphate 14 in 18\% yield. Sulfo-Cy5 was coupled to this molecule using its NHS ester to give triphosphate $\mathbf{1 5}$ in a yield of $72 \%$. The azide of compound 15 was reduced using TCEP to give $80 \%$ of compound $\mathbf{1 6}$ bearing the free amine. Coupling of the Sulfo-Cy3 NHS ester gave the O3' and $\gamma$-labelled probe 17 in $61 \%$ yield.
We also studied these analogues in the SVPD assay as described above (Fig. 3). Both combinations of attachment sites used in this case resulted in high changes of the ratio of fluorescence intensities that albeit differed with a 77-fold change upon cleavage for compound 13 (Fig. 3A, ESI Fig. S6†) and an approx. 120-fold change observed for compound 17 (Fig. 3B, ESI Fig. S7†). These changes are in the same range as seen for the analogous $O 2^{\prime}$-modified derivative. These analogues broaden the repertoire of doubly labelled ATP analogues that can be used to study ATP cleavage. This also demonstrates the general applicability of the combination of Sulfo-Cy3 and Sulfo-Cy5 for probes of this type of molecules.

\section{Conclusion}

In conclusion, we present the synthesis of seven different new doubly dye labelled ATP analogues. In the synthesis strategy, we utilize a trifluoroacetamide and an azide as amine precursors to build up the doubly modified ATP scaffolds. The trifluoroacetamide was easily deprotected to the free amine using alkaline conditions without affecting the azide. After coupling of the first dye using NHS ester chemistry, the azide was converted to an amine without affecting the dyes exploiting the mild conditions of the Staudinger reaction. Finally, the second dye was attached using NHS ester chemistry resulting in five different doubly labelled ATP analogues modified at the $O 2^{\prime}$ - and the $\gamma$-position. Thus, this synthesis strategy turned 
A

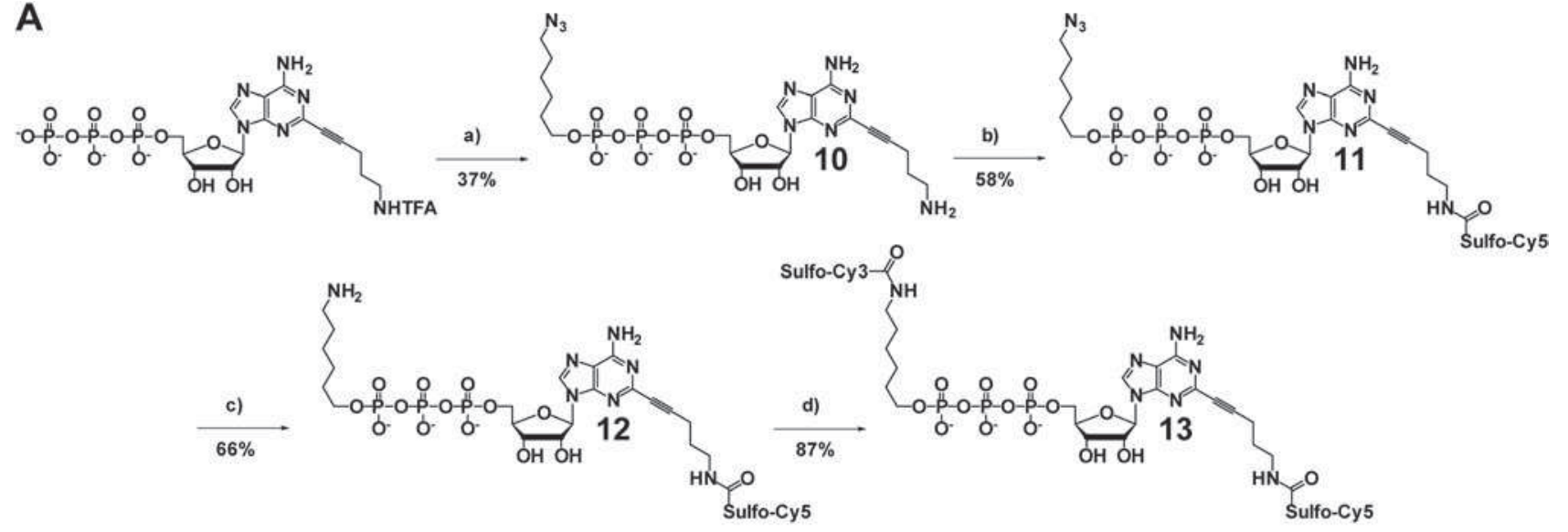

B

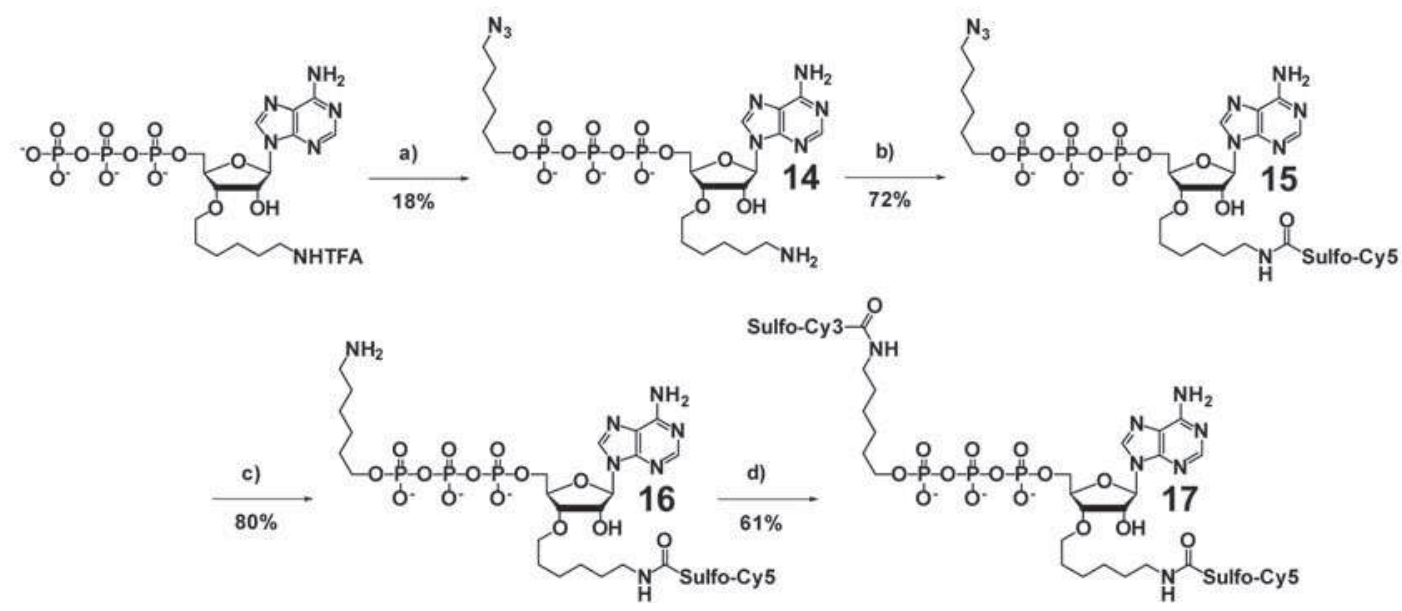

Scheme 3 Synthesis of ATP analogues doubly labelled at the $\gamma$ phosphate and the C2 (13) or O3' position (17), respectively. (a) (i) 6 Azido 1 bromohexane, mole cular sieves, DMF, r.t., 12 h; (ii) ammonia in water, r.t., 2 h (10) or 4 h (14); (b) Sulfo Cy5 NHS ester, DMF, carbonate buffer (pH 8.9 ), r.t., 12 h; (c) TCEP HCl, Et 3 N, methanol, water, r.t., $4 \mathrm{~h} \mathrm{(12)}$ or $12 \mathrm{~h} \mathrm{(16);} \mathrm{(d)} \mathrm{Sulfo} \mathrm{Cy3} \mathrm{NHS} \mathrm{ester,} \mathrm{DMF,} \mathrm{carbonate} \mathrm{buffer} \mathrm{(pH} \mathrm{8.9),} \mathrm{r.t.,} 12$ h. All compounds were prepared as their triethylammo nium salts.

A

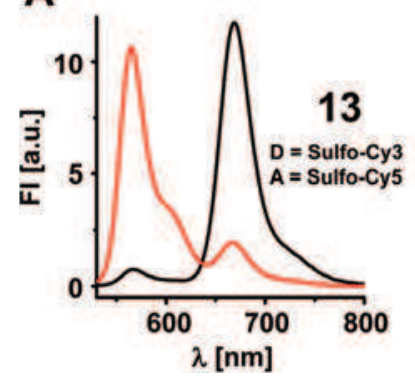

B

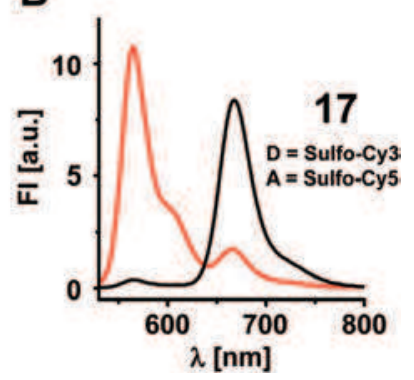

Fig. 3 Fluorescence spectra of ATP analogues doubly labelled at the $\gamma$ phos phate and the C2 (13) or $03^{\prime}$ position (17), respectively, after incubation without (black) or with (red) the phosphodiesterase I of C. adamanteus measured at a concentration of $1 \mu \mathrm{M}$ in $1 \times$ PBS with excitation at $510 \mathrm{~nm}$.

out to be a highly flexible approach towards customized, doubly labelled nucleotides that can be used to visualize their cleavage.

We further investigated the fluorescent characteristics of these probes using SVPD as a model enzyme. We tested different combinations of dyes that may be used to construct FRET cassettes. We found two combinations of two fluorescent dyes, Sulfo-Cy3 and Sulfo-Cy5 as well as Cy3 and Cy5, that can be used to construct ratiometric FRET sensors for ATP cleavage with changes of fluorescence ratios of up to 140-fold. Furthermore, using Sulfo-Cy3 and the Eclipse quencher we were able to construct a dark quenched ATP analogue, whose fluorescence rises 66-fold upon cleavage.

To expand the scope of doubly labelled ATP analogues we also synthesized ATP analogues modified at the $C 2$ - and the $O 3^{\prime}$-position as well as the $\gamma$-phosphate. Both analogues containing the combination of Sulfo-Cy3 and Sulfo-Cy5 were synthesized in a straight-forward manner using the established synthesis protocols. Comparable changes in fluorescence characteristics upon cleavage as seen for the $O 2$ '-modified analogue were observed for both analogues. This shows that these probes can also efficiently be used to monitor ATP cleavage. Furthermore, it demonstrates that the combination of Sulfo- 
Cy3 and Sulfo-Cy5 is generally applicable for this kind of probes.

The presented FRET based activity probes clearly demonstrate the high potential of this concept to monitor ATP consuming enzymes by the vast changes of the easily detectable fluorescence signal.

\section{Experimental section}

\section{SVPD assay}

The corresponding doubly dye labelled ATP analogue $(100 \mu \mathrm{M})$ was processed with or without SVPD $\left(2 \mu \mathrm{g} \mathrm{ml} \mathrm{m}^{-1}\right)$ in a buffer containing $100 \mathrm{mM} \mathrm{NaCl}, 50 \mathrm{mM}$ Tris- $\mathrm{HCl}$ (pH 7.9), $10 \mathrm{mM}$ $\mathrm{MgCl}_{2}$ and $1 \mathrm{mM} \mathrm{DTT}$ in a total volume of $60 \mu \mathrm{l}$ for $30 \mathrm{~min}$ at $30{ }^{\circ} \mathrm{C}$. In the cases of the least polar analogues $7 \mathbf{c}$ and $7 \mathrm{e}$ these conditions did not result in complete cleavage as precipitation of the probes occurred. Therefore, probe $7 \mathrm{c}(10 \mu \mathrm{M})$ was treated with $16 \mu \mathrm{g} \mathrm{ml}^{-1}$ SVPD in a buffer containing $50 \mathrm{mM}$ $\mathrm{NaCl}, 25 \mathrm{mM}$ Tris-HCl (pH 7.9), $5 \mathrm{mM} \mathrm{MgCl}$ and $500 \mu \mathrm{M}$ DTT in a total volume of $600 \mu \mathrm{l}$ for $30 \mathrm{~min}$ at $30{ }^{\circ} \mathrm{C}$. Probe $7 \mathrm{e}$ $(10 \mu \mathrm{M})$ was treated for $2 \mathrm{~h}$ at $30^{\circ} \mathrm{C}$ using the same conditions as for $7 \mathbf{c}$ with the exception that $10 \%$ DMSO were used in the buffer. All reactions were quenched by the addition of $60 \mu \mathrm{l}$ 0.1 M EDTA.

\section{Fluorescence measurements}

For fluorescence measurements the SVPD reactions were diluted to a final concentration of the probe of $1 \mu \mathrm{M}$ with $1 \times$ PBS buffer and the fluorescence spectra of the crude reaction solutions were measured. Excitation of Sulfo-Cy3 and Cy3 was performed using a wavelength of $510 \mathrm{~nm}$. Sulfo-Cy5 was excited at $610 \mathrm{~nm}$. Fluorescence intensities were determined by integration of the fluorescence spectra from $540 \mathrm{~nm}$ to $620 \mathrm{~nm}(640 \mathrm{~nm}$ to $720 \mathrm{~nm}$ for $7 \mathbf{b}$ ) for donor fluorescence intensity and from $640 \mathrm{~nm}$ to $720 \mathrm{~nm}$ for acceptor fluorescence intensity (7a, 7c, 13 and 17). For quenched ATP analogues (7b, $\mathbf{7 d}$, and $7 \mathrm{e}$ ) the change of fluorescence intensity was calculated as the quotient of the donor fluorescence intensity in the cleaved and in the non-cleaved states. For ratiometric FRET probes (7a, 7c, 13 and 17) the ratio of fluorescence intensities was calculated as the quotient of the donor and the acceptor fluorescence intensities. Change of the ratio of fluorescence intensities was calculated as the quotient of the ratio of fluorescence intensities in the cleaved and in the non-cleaved states.

\section{RP-HPLC and ESI-MS analysis}

For HPLC analyses the SVPD reactions were separated by analytical RP-HPLC (a linear gradient of acetonitrile in $50 \mathrm{mM}$ aqueous triethylammonium acetate (TEAA buffer, $\mathrm{pH}$ 7.0) was used). Fractions containing the ATP analogues or their fragments were collected and further characterized by ESI-MS.

\section{Acknowledgements}

We gratefully acknowledge the funding by the Deutsche Forschungsgemeinschaft within the SFB 969, the Konstanz Research School Chemical Biology for a stipend to N. H., the Studienstiftung des deutschen Volkes and the Zukunftskolleg of the University of Konstanz for a stipend to S. M. H.

\section{Notes and references}

1 (a) J. R. Knowles, Annu. Rev. Biochem., 1980, 49, 877; (b) R. D. Vale and R. A. Milligan, Science, 2000, 288, 88; (c) W. Junge, H. Sielaff and S. Engelbrecht, Nature, 2009, 459, 364; (d) J. F. Allen, Cell, 2002, 110, 273.

2 (a) A. Kornberg, J. F. Scott and L. L. Bertsch, J. Biol. Chem., 1978, 253, 3298; (b) H. Wegele, P. Muschler, M. Bunck, J. Reinstein and J. Buchner, J. Biol. Chem., 2003, 278, 39303; (c) T. Sinkunas, G. Gasiunas, C. Fremaux, R. Barrangou, P. Horvath and V. Siksnys, EMBO J., 2011, 30, 1335; (d) C. J. Hastie, H. J. McLauchlan and P. Cohen, Nat. Protoc., 2006, 1, 968.

3 (a) T. P. Geladopoulos, T. G. Sotiroudis and A. E. Evangelopoulos, Anal. Biochem., 1991, 192, 112; (b) M. Catarinella, T. Grüner, T. Strittmatter, A. Marx and T. U. Mayer, Angew. Chem., Int. Ed., 2009, 48, 9072; (c) C. E. Berndsen and C. Wolberger, Anal. Biochem., 2011, 418, 102.

4 (a) M. A. Woo, in Methods in Molecular Biology, ed. N. Osheroff and M. A. Bjornsti, Humana Press Inc., 1999, vol. 95, p. 311; (b) T.-G. Huang and D. D. Hackney, J. Biol. Chem., 1994, 269, 16493.

5 S. M. Hacker, D. Pagliarini, T. Tischer, N. Hardt, D. Schneider, M. Mex, T. U. Mayer, M. Scheffner and A. Marx, Angew. Chem., Int Ed., 2013, DOI: 10.1002/anie.201304723.

6 B. A. Schulman and J. W. Harper, Nat. Rev. Mol. Cell Biol., 2009, 10, 319.

7 (a) J. J. Allen, et al., Nat. Methods, 2007, 4, 511; (b) J. D. Blethrow, J. S. Glavy, D. O. Morgan and K. M. Shokat, Proc. Natl. Acad. Sci. U. S. A., 2008, 105, 1442; (c) K. Shah, Y. Liu, C. Deirmengian and K. M. Shokat, Proc. Natl. Acad. Sci. U. S. A., 1997, 94, 3563; (d) L. M. Elphick, S. E. Lee, V. Gouverneur and D. J. Mann, ACS Chem. Biol., 2007, 2, 299; (e) T. M. Kapoor and T. J. Mitchison, Proc. Natl. Acad. Sci. U. S. A., 1999, 96, 9106.

8 S. M. Hacker, N. Hardt, A. Buntru, D. Pagliarini, M. Möckel, T. U. Mayer, M. Scheffner, C. R. Hauck and A. Marx, Chem. Sci., 2013, 4, 1588.

9 (a) T. Hiratsuka, Biochim. Biophys. Acta, 1983, 742, 496; (b) C. T. Friel and J. Howard, EMBO J., 2011, 30, 3928; (c) J. C. Wolters, G. Roelfes and B. Poolman, Bioconjugate Chem., 2011, 22, 1345; (d) T. Kamei, T. Fukaminato and N. Tamaoki, Chem. Commun., 2012, 48, 7625; (e) J. Q. Cheng, W. Jiang and D. D. Hackney, Biochemistry, 1998, 37, 5288; $(f)$ D. Thoenges, E. Amler, T. Eckert and W. Schoner, J. Biol. Chem., 1999, 274, 1971. 
10 S. M. Hacker, M. Mex and A. Marx, J. Org. Chem., 2012, 77, 10450.

11 R. B. Mujumdar, L. A. Ernst, S. R. Mujumdar, C. J. Lewis and A. S. Waggoner, Bioconjugate Chem., 1993, 4, 105.

12 A. Dietrich, V. Buschmann, C. Müller and M. Sauer, Rev. Mol. Biotechnol., 2002, 82, 211.

13 M. A. G. Sillero, O. Madrid, E. Zaera and A. Sillero, Biochim. Biophys. Acta, 1997, 1334, 191.

14 H. Staudinger and J. Meyer, Helv. Chim. Acta, 1919, 2, 635.

15 P. Ranjitkar, B. G. K. Perera, D. Swaney, S. B. Hari, E. T. Larson, R. Krishnamurty, E. A. Merritt, J. Villén and D. J. Maly, J. Am. Chem. Soc., 2012, 134, 19017.
16 (a) S. Takahashi, W. Piao, Y. Matsumura, T. Komatsu, T. Ueno, T. Terai, T. Kamashi, M. Kohno, T. Nagano and K. Hanaoka, J. Am. Chem. Soc., 2012, 134, 19588; (b) X. Peng, Z. Yang, J. Wang, J. Fan, Y. He, F. Song, B. Wang, S. Sun, J. Qu, J. Qi and M. Yan, J. Am. Chem. Soc., 2011, 133, 6626; (c) K. Kiyose, K. Hanaoka, D. Oushiki, T. Nakamura, M. Kajimura, M. Suematsu, H. Nishimatsu, T. Yamane, T. Terai, Y. Hirata and T. Nagano, J. Am. Chem. Soc., 2010, 132, 15846.

17 J. M. Heemstra and J. S. Moore, Tetrahedron, 2004, 60, 7287. 